\title{
Combined Fecal Transferrin Test and Immuno Fecal Occult Blood Test for Detecting Colorectal Cancer and Advanced Adenoma in Asymptomatic and Symptomatic Populations
}

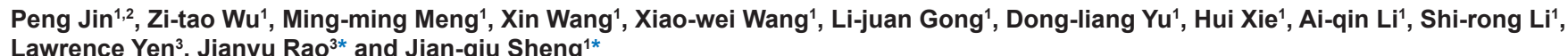

${ }^{1}$ Department of Gastroenterology, Beijing Military General Hospital, Beijing, People's Republic of China

${ }^{2}$ Postgraduate School, Third Military Medical University, Chongqing, People's Republic of China

${ }^{3}$ Department of Pathology and Laboratory Medicine, David Geffen School of Medicine and Department of Epidemiology, School of Public Health, University of California, Los Angeles, California, USA

\begin{abstract}
Recent proteomic studies identified Transferin (TF) as a potential colon cancer biomarker. A dipstick TF test similar to Immuno Fecal Occult Blood Test (IFOBT) was developed, and an initial study showed the TF test had compatible performance characteristics for detecting colon cancer and adenoma. The goal of this study was to evaluate the efficacy of the combination of TF and IFOBT for detecting advanced adenomas and cancer in asymptomatic and symptomatic populations. A total of 1,943 healthy subjects (asymptomatic group) and 201 subjects with various gastrointestinal symptoms (symptomatic group) were recruited for the study. For asymptomatic subjects, one fecal sample was collected for concurrent TF and IFOBT testing. Colonoscopy was performed for individuals positive for either TF or IFOBT. For the symptomatic subjects, each individual underwent TF, IFOBT, and colonoscopy simultaneously. For asymptomatic group, 1,737 individuals tested for TF and IFOBT, 251 subjects (14.5\%) showed either TF or IFOBT positivity. Colonoscopy was performed for 193 of the 251 individuals. A total of 3 colorectal cancers and 43 advanced adenomas were detected. Combination of the two tests (either/or) significantly increased the detection rate for colorectal cancers and advanced adenomas compared to IFOBT alone ( $2.6 \%$ vs. $1.6 \%, P=0.034)$. In the symptomatic group, the combined test also significantly increased the sensitivity for detecting advanced adenomas and cancer than that of IFOBT alone $(77.9 \%$ vs. $55.9 \%, P=0.006)$, but with decreased specificity $(42.1 \%$ vs $63.9 \%, P=0.005)$. Combined TF and IFOBT test increased the detection rate of colorectal adenoma and cancer in both asymptomatic and symptomatic populations.
\end{abstract}

Keywords: Colorectal cancer screen; Transferrin; Immuno fecal occult blood test

\section{Introduction}

The incidence of Colorectal Cancer (CRC) has increased rapidly in China and continues to be a major public health threat around the world. In Beijing, the annual incidence of CRC increased from 16 to 24 per 100,000 in the past decade [1,2]. There is ample evidence to support that screening and early detection reduce the mortality of colorectal cancer. Fecal occult blood test, especially the guaiac-based (gFOBT) test, is widely used in colorectal cancer screening and has been considered to be the preferred method in countries where medical resources are limited [3]. However, the sensitivity of FOBT is rather low. Antibody based Immuno Fecal Occult Blood Test (IFOBT) method improved the sensitivity, specificity, and cost-effectiveness of colorectal cancer screening [4-6], however still about 20 to $40 \%$ colorectal cancers and 50 to $70 \%$ advanced adenomas may be missed with one-time IFOBT testing $[5,6]$.

Transferrin, a type of $\beta 1$ globulin with a molecular weight of $77 \mathrm{KD}$, is responsible for transferring extracellular iron into cells through cell membrane receptor-mediated endocytosis [7]. Also, transferrin is more stable than hemoglobin $[8,9]$. When gastrointestinal bleeding occurs, serum transferrin enters the gastrointestinal tract and is excreted in feces. Previous proteomic studies identified transferrin as a potential protein marker for a number of epithelial cancers, including colorectal cancer [10]. Recently, we performed a dipstick based transferrin test (fecal transferrin test, or TF) in conjunction with IFOBT in a crosssectional study involving clinical patient samples. Our preliminary study in a small group of colorectal cancer, precancerous adenoma, and normal control stool samples showed that the sensitivity of TF plus IFOBT for detecting colon cancer and precancerous lesions was significantly higher than that of IFOBT alone [11]. In the current study, we further evaluated the efficacy of one-time point TF combined with IFOBT test for the detection of colorectal cancers and precancerous lesions in both routine screening (asymptomatic population) and clinical (symptomatic population) settings in a prospective manner.

\section{Materials and Methods}

\section{Subjects}

This study involved two groups of Beijing residents. The first group included 1,943 retired military personnel, ages 50 to 75 , all were generally in good health, without gastrointestinal symptoms, and had no history of colorectal cancer, colorectal adenomas, inflammatory

*Corresponding authors: Jianyu Rao, Department of Pathology and Laboratory Medicine, David Geffen School of Medicine, and Department of Epidemiology, School of Public Health, University of California, Los Angeles, CA, USA, Tel: $310-$ 794-1567; Fax: 310-825-7795; E-mail: jrao@mednet.ucla.edu

Jian-qiu Sheng, Department of Gastroenterology, Beijing Military General Hospital, Nanmenchang 5\#, Dongcheng District, Beijing, China, 100700, E-mail: jianqiu@263.net

Received July 30, 2012; Accepted August 06, 2012; Published August 08, 2012

Citation: Jin P, Wu ZT, Meng MM, Wang X, Wang XW, et al. (2012) Combined Fecal Transferrin Test and Immuno Fecal Occult Blood Test for Detecting Colorectal Cancer and Advanced Adenoma in Asymptomatic and Symptomatic Populations. J Cancer Sci Ther 4: 243-248. doi:10.4172/1948-5956.1000149

Copyright: (c) 2012 Jin P, et al. This is an open-access article distributed under the terms of the Creative Commons Attribution License, which permits unrestricted use, distribution, and reproduction in any medium, provided the original author and source are credited. 
Citation: Jin P, Wu ZT, Meng MM, Wang X, Wang XW, et al. (2012) Combined Fecal Transferrin Test and Immuno Fecal Occult Blood Test for Detecting Colorectal Cancer and Advanced Adenoma in Asymptomatic and Symptomatic Populations. J Cancer Sci Ther 4: $243-248$. doi:10.4172/1948-5956.1000149

bowel disease or malignant tumors. All subjects have been receiving annual screening as part of a welfare program for veterans. Among the eligible subjects, 1,737 individuals agreed to participate in this study. Of the 1,737 participants, 1,524 were men and 213 were women, with a mean age of 62.2 years old. The study period was from May 2010 to December 2010.

The second group included Beijing Military General Hospital patients seen between October 2009 and March 2010. The inclusion criteria were bloody stool (at least once), changes in bowel habits and evacuation, unexplained weight loss, anemia, and abdominal pain in the past 4 weeks. Patients with known history of inflammatory bowel disease or colorectal adenomas or carcinomas were excluded from the study, giving a total of 201 eligible subjects. Of these, 153 were men and 48 were women, with a mean age of 67.0 years old (ranging from 31-91).

The study was approved by the ethic committee of the Beijing Military General Hospital.

\section{TF and IFOBT}

For individuals in the asymptomatic group, each subject provided a stool sample that was used for TF and IFOBT testing. Diet and medication were not restricted in the subjects before providing the stool sample. The IFOBT kits were provided by Hemosure Inc.(Irwindale, CA, USA) and the TF test kits were provided by WHPM, Inc. (EI Monte, CA, USA). The tests were performed according to the manufacturers instructions. The positive threshold of IFOBT (gold gel stripe) and TF was $0.2 \mu \mathrm{g} / \mathrm{ml}$ and $30 \mathrm{ng} / \mathrm{ml}$, respectively. Individuals positive for either IFOBT and/or TF were asked to undergo colonoscopy.

For each individual in the symptomatic group, a stool sample was collected for the TF and IFOBT tests, performed in a similar fashion as the asymptomatic group. The colonoscopy was performed on the next day regardless of the findings of the TF and IFOBT tests.

\section{Colonoscopy}

Colonoscopy was performed by experienced gastroenterologists

\begin{tabular}{|c|c|c|c|c|c|c|c|}
\hline & IFOBT & TF & Only IFOBT & Only TF & ВОTH & Either & $P$ Value $^{f}$ \\
\hline Positive patients ${ }^{a}$ & $139(8.0)$ & 184(10.6) & $67(3.9)$ & $112(6.4)$ & $72(4.1)$ & $251(14.5)$ & 0.000 \\
\hline Colonoscopy performed ${ }^{b}$ & $109(78.4)$ & $143(77.7)$ & $50(74.6)$ & $84(75.0)$ & $59(81.9)$ & 193(76.9) & 0.730 \\
\hline \multicolumn{8}{|l|}{ Detection rate $^{c}$} \\
\hline All advanced adenomas and cancer & $28(1.6)$ & $31(1.8)$ & $15(0.9)$ & $18(1.0)$ & $13(0.7)$ & $46(2.6)$ & 0.034 \\
\hline Cancer & $2(0.1)$ & $2(0.1)$ & $1(0.1)$ & $1(0.1)$ & $1(0.1)$ & $3(0.2)$ & 0.654 \\
\hline Advanced adenoma $\geq 10 \mathrm{~mm}$ & $24(1.4)$ & $22(1.3)$ & $13(0.7)$ & $11(0.6)$ & $11(0.6)$ & $35(2.0)$ & 0.149 \\
\hline Advanced adenoma $<10 \mathrm{~mm}^{\mathrm{d}}$ & $2(0.1)$ & $7(0.4)$ & $1(0.1)$ & $6(0.3)$ & $1(0.1)$ & $8(0.5)$ & 0.057 \\
\hline \multicolumn{8}{|l|}{ Positive predictive value ${ }^{e}$} \\
\hline All advanced adenomas and cancer & $28(25.7)$ & $31(21.7)$ & $15(30)$ & $18(21.4)$ & $13(22.0)$ & $46(23.8)$ & 0.719 \\
\hline Cancer & $2(1.8)$ & $2(1.4)$ & $1(2.0)$ & $1(1.2)$ & $1(1.7)$ & $3(1.6)$ & 0.854 \\
\hline Advanced adenoma $\geq 10 \mathrm{~mm}$ & $24(22.0)$ & $22(15.4)$ & $13(26.0)$ & $11(13.1)$ & $11(18.6)$ & $35(18.1)$ & 0.414 \\
\hline Advanced adenoma $<10 \mathrm{~mm}{ }^{\mathrm{d}}$ & $2(1.8)$ & $7(4.9)$ & $1(2.0)$ & $6(7.1)$ & $1(1.7)$ & $8(4.1)$ & 0.281 \\
\hline
\end{tabular}

a Rates are the number of positive patients relative to the number of participants.

b Rates are the number of colonoscoped patients relative to the number of positive patients.

c Detection rate per protocol is the percentage of persons with lesions relative to the number of participants.

$\mathrm{d} \geq 1$ villous adenoma or adenoma with high-grade dysplasia and no adenomas $\geq 10 \mathrm{~mm}$

e PPV is the number of true positives relative to the number of total positive patients. followed up with colonoscopy.

f TF combined with IFOBT (Either/or) vs IFOBT alone.

Table 1: Results of IFOBT and TF, either alone or combination, and subsequent colonoscopic findings in asymptomatic population (1737 participants), n(\%).

\begin{tabular}{|c|c|c|c|c|c|c|}
\hline & Men $(n=1524)$ & Women $(n=213)$ & $P$ value & Age $<60(n=452)$ & Age $\geq 60(n=1285)$ & $P$ value \\
\hline \multicolumn{7}{|c|}{ Test Results ${ }^{a}$} \\
\hline IFOBT & $128(8.4)$ & $11(5.2)$ & 0.103 & $31(6.9)$ & $108(8.4)$ & 0.297 \\
\hline TF & $153(10.0)$ & $31(14.6)$ & 0.045 & $46(10.2)$ & $138(10.7)$ & 0.738 \\
\hline BOTH & $65(4.3)$ & $7(3.3)$ & 0.502 & $15(3.3)$ & $57(4.4)$ & 0.305 \\
\hline EITHER & $216(14.2)$ & $35(16.4)$ & 0.380 & $62(13.7)$ & $189(14.7)$ & 0.606 \\
\hline \multicolumn{7}{|c|}{ Colonoscopy ${ }^{b}$} \\
\hline IFOBT & $102(79.7)$ & $7(63.6)$ & 0.214 & $15(48.4)$ & $94(87.0)$ & 0.000 \\
\hline TF & $125(81.7)$ & $18(58.1)$ & 0.004 & $29(63.0)$ & $114(82.6)$ & 0.006 \\
\hline BOTH & $54(83.1)$ & $5(71.4)$ & 0.446 & $7(46.7)$ & $52(91.2)$ & 0.000 \\
\hline EITHER & $173(80.1)$ & $20(57.1)$ & 0.003 & $37(59.7)$ & $156(82.5)$ & 0.000 \\
\hline \multicolumn{7}{|c|}{ Detection rate ${ }^{c}$} \\
\hline \multicolumn{7}{|c|}{ All advanced adenomas and cancer } \\
\hline IFOBT & $27(1.8)$ & $1(0.5)$ & 0.158 & $1(0.2)$ & $27(2.1)$ & 0.006 \\
\hline TF & $30(2.0)$ & $1(0.5)$ & 0.122 & $4(0.9)$ & $27(2.1)$ & 0.093 \\
\hline BOTH & $12(0.8)$ & $1(0.5)$ & 0.614 & $1(0.2)$ & $12(0.9)$ & 0.131 \\
\hline EITHER & $45(3.0)$ & $1(0.5)$ & 0.034 & $4(0.9)$ & $42(3.3)$ & 0.007 \\
\hline
\end{tabular}

a Rates are the number of positive patients relative to the number of participants per group.

$b$ Rates are the number of colonoscoped patients relative to the number of positive patients per group.

c Detection rate per protocol is the percentage of persons with lesions relative to the number of participants per group.

Table 2: Results of tests and subsequent colonoscopic findings in asymptomatic population stratified by gender and age, $\mathrm{n}(\%)$ 
Citation: Jin P, Wu ZT, Meng MM, Wang X, Wang XW, et al. (2012) Combined Fecal Transferrin Test and Immuno Fecal Occult Blood Test for Detecting Colorectal Cancer and Advanced Adenoma in Asymptomatic and Symptomatic Populations. J Cancer Sci Ther 4: $243-248$. doi:10.4172/1948-5956.1000149

\begin{tabular}{|c|c|}
\hline Clinical features & No. of patients \\
\hline Changes in bowel habit & $127(63.2 \%)$ \\
\hline Bloody stool & $89(44.3 \%)$ \\
\hline Abdominal pain & $74(36.8 \%)$ \\
\hline Weight loss & $46(22.9 \%)$ \\
\hline Anemia & $41(20.4 \%)$ \\
\hline Total & 201 \\
\hline
\end{tabular}

Table 3: The distribution of clinical features in symptomatic population ( $n=201)$

(PJ, JQS, and SRL), who were blinded to the TF and IFOBT test results. All colonoscopies reached the ileocecal junction. In addition, biopsies were performed in all lesions that were identified by colonoscopy and reviewed by pathology. Villous adenoma (at least $25 \%$ villous), adenoma with high-grade dysplasia, or adenomas with a diameter $\geq 10 \mathrm{~mm}$ were regarded as advanced adenomas. If more than 1 lesion was present, a patient was classified by the most advanced lesion. The identified CRC patients underwent surgical treatment in Beijing Military General Hospital, and Dukes staging on resected specimens was determined.

\section{Statistical analysis}

Detection rates and positive predictive values of IFOBT alone, TF alone, and TF combined with IFOBT for colorectal cancer and advanced adenomas were calculated. Moreover, sensitivity, specificity, positive predictive value, and negative predictive values were calculated in the symptomatic group as all of the patients underwent colonoscopy. The chi square test was used to analyze the significance of difference. Statistical significance was established at $P<0.05$. SPSS 13.0 software was used for statistical analyses.

\section{Results}

In the asymptomatic group, 1,737 individuals consented and underwent both TF and IFOBT tests. As shown in Table 1, IFOBT was positive in 139 individuals $(8.0 \%)$, while TF was positive in 184 individuals (10.6\%), totaling up to 251 individuals (14.5\%) who were positive for TF and/or IFOBT. Of these 251 individuals, 193 (76.9\%) consented to receive colonoscopy; among them 43 individuals were found with advanced adenomas, and 3 individuals were diagnosed with colorectal cancer ( 1 in Dukes stage A and 2 in stage B). IFOBTpositive subjects identified 2 carcinomas ( 2 in stage $B$ ) and 26 advanced adenomas for a $1.6 \%$ (28 of 1,737$)$ detection rate, which was similar to the $1.8 \%$ (31 of 1,737$)$ in TF-positive individuals with 2 carcinomas ( 1 in stage A and 1 in stage B) and 29 advanced adenomas. Compared with IFOBT alone, the detection rate of TF plus IFOBT in either/or scenario for colorectal cancer and advanced adenomas was significantly increased $(2.6 \%, 46$ of 1,737 versus $1.6 \%, 28$ of $1,737, P=0.034)$. The most notable increase was seen in the subgroup of advanced adenomas $<10 \mathrm{~mm}$, though the difference did not reach statistical significance ( 8 of 1737 versus 2 of 1737, $P=0.057$ ). The difference for positive predictive value was similar between TF plus IFOBT and IFOBT alone for the detection of carcinoma and advanced adenomas (25.7\% versus $23.8 \%, P=0.719$ ).

Table 2 presents the test results and subsequent colonoscopy findings stratified by sex (male versus female) and age ( $<60$ versus $>=60$ ). Overall there were more women and younger subjects among those who refused colonoscopy than those who underwent colonoscopy $(25.9 \%, 15 / 58$ and $43.1 \%, 25 / 58$ versus $10.4 \%, 20 / 193$ and $19.2 \%, 37 / 193, P=0.003$ and 0.000 , respectively). The positive rate of TF alone was higher among women than among men (14.6\% versus $10.0 \%$, $P=0.045)$. However, when TF was combined with IFOBT in either/or setting, the difference for positive rate was not significant between men and women ( $14.2 \%$ versus $16.4 \%, P=0.380)$. The detection rate for advanced adenomas and cancer by IFOBT was higher among individuals over 60 years old $(P=0.006)$ and by TF combined with IFOBT was higher among men and older age group than women and younger age group ( $P=0.034$ and 0.007 , respectively).

We also tested TF and IFOBT in a group of patients with various gastrointestinal symptoms $(n=201)$ who were admitted to our clinic as a part of routine checkup. Table 3 shows the overall clinical presentation of these patients. In this symptomatic group, 21 colorectal cancers (10.4\%) and 47 advanced adenomas (23.4\%) were diagnosed by colonoscopy with histopathology (Table 4). Among 21 CRC patients, there was 1 case of Dukes stage A, 8 cases of stage B, 11 cases of stage $\mathrm{C}$ and 1 case of stage D. Positivity for either IFOBT and/or TF was identified in 130 of the symptomatic individuals. The sensitivities of IFOBT, TF, and TF plus IFOBT for colorectal cancer were $57.1 \%$ (12 of 21 ), $76.2 \%$ (16 of 21 ), and $85.7 \%$ (18 of 21 ), respectively. The sensitivity of TF in conjunction with IFOBT for colorectal cancer and advanced adenomas was significantly higher than that of IFOBT alone (77.9\% versus $55.9 \%, P=0.006$ ), while the specificity was significantly lower $(42.1 \%$ versus $63.9 \%, P=0.005)$ and positive and negative predictive values showed no significant differences $(P=0.619$ and 0.443 , respectively).

Table 5 shows the results of tests and subsequent colonoscopic findings in symptomatic population stratified by gender and age. The positive rate of TF alone was higher in females $(P=0.024)$. However, no significant differences of sensitivity for detecting adenoma and cancer were seen between different sex and age groups with different tests. Table 6 compared the sensitivity/specificity of IFOBT versus IFOBT combined with TF in patients with three main symptoms (changes in bowel habits, bloody stool, and abdominal pain). There was a significant increase of sensitivity in detecting advanced adenoma and cancer in all three categories. As expected, there was a concomitant significant decrease of specificity in patients with changes in bowel habits and abdominal pain but not in patients with bloody stool.

\section{Discussion}

For colorectal cancer screening, FOBT, especially IFOBT, is widely accepted as an effective method. However, FOBT has limited sensitivity due to the instability of hemoglobin. Moreover, FOBT may not be able to detect non-hemorrhagic early lesions. Therefore, the sensitivity of FOBT for the detection of colorectal cancer, especially advanced adenomas, is rather poor (ranging from $27-66 \%$ with one sample test) $[5,6]$. In the past few years, other fecal-based biological markers have also been developed in addition to FOBT. However, many of these markers, such as fecal micro-albumin [12] and fecal calprotectin [13], failed to improve the detection rate of colorectal cancers. While the sensitivity of the stool DNA test is higher than FOBT, the improvements offered by this method are still limited (52\% for invasive cancer and $41 \%$ for cancer and adenoma with high-grade dysplasia) [14]. More importantly, the high cost of DNA based screening tests limits its feasibility in developing countries with limited resources.

Transferrin accounts for about $0.3 \%-0.5 \%$ of plasma protein and is mainly synthesized in the liver. Transferrin transfers extracellular iron into cells through cell membrane receptor-mediated endocytosis, and each transferrin molecule can combine with the presence of two iron atoms. Furthermore, transferrin is essential for cell growth and survival. Like hemoglobin, transferrin enters stool through bleeding; however, unlike hemoglobin, transferrin is more stable and less affected by 
Citation: Jin P, Wu ZT, Meng MM, Wang X, Wang XW, et al. (2012) Combined Fecal Transferrin Test and Immuno Fecal Occult Blood Test for Detecting Colorectal Cancer and Advanced Adenoma in Asymptomatic and Symptomatic Populations. J Cancer Sci Ther 4: $243-248$. doi:10.4172/1948-5956.1000149

\begin{tabular}{|c|c|c|c|c|c|c|c|}
\hline & IFOBT & TF & Only IFOBT & Only TF & ВотH & EITHER & $P$ value $^{\text {a }}$ \\
\hline Positive patients ${ }^{\mathrm{b}}$ & $86(42.8)$ & 93(46.3) & $37(18.4)$ & $44(21.9)$ & $49(24.4)$ & $130(64.7)$ & 0.000 \\
\hline \multicolumn{8}{|l|}{ Sensitivity ${ }^{c}$} \\
\hline All advanced adenomas and cancer(68) & $38(55.9)$ & $40(58.8)$ & 13(19.1) & $15(22.1)$ & $25(36.8)$ & $53(77.9)$ & 0.006 \\
\hline Cancer(21) & $12(57.1)$ & $16(76.2)$ & $2(9.5)$ & $6(28.6)$ & $10(47.6)$ & 18(85.7) & 0.040 \\
\hline Dukes A/B Cancer(9) & $4(44.4)$ & $6(66.7)$ & 1(11.1) & 3(33.3) & $3(33.3)$ & $7(77.8)$ & 0.147 \\
\hline Dukes C/D Cancer(12) & $8(66.7)$ & $10(83.3)$ & $1(8.3)$ & $3(25.0)$ & $7(58.3)$ & 11(91.7) & 0.142 \\
\hline Advanced adnomas(47) & $26(55.3)$ & $24(51.1)$ & 11(23.4) & $9(19.1)$ & 15(31.9) & $35(74.5)$ & 0.052 \\
\hline Advanced adenoma $\geq 10 \mathrm{~mm}(36)$ & 21(58.3) & $16(44.4)$ & $10(27.8)$ & $5(13.9)$ & 11(30.6) & $26(72.2)$ & 0.216 \\
\hline Advanced adenoma $<10 \mathrm{~mm}(11)^{d}$ & $5(45.5)$ & $8(72.7)$ & $1(9.1)$ & $4(36.4)$ & $4(36.4)$ & $9(81.8)$ & 0.076 \\
\hline \multicolumn{8}{|l|}{ e } \\
\hline All advanced adenomas and cancer(68) & 48(63.9) & $53(60.2)$ & $24(82.0)$ & $29(78.2)$ & $24(82.0)$ & $77(42.1)$ & 0.005 \\
\hline Cancer(21) & 74(58.9) & $77(57.2)$ & $35(80.6)$ & $38(78.9)$ & $39(78.3)$ & 112(37.8) & 0.005 \\
\hline \multicolumn{8}{|l|}{ Negative predictive value ${ }^{f}$} \\
\hline All advanced adenomas and cancer(68) & $85(73.9)$ & $80(74.1)$ & $109(66.5)$ & 104(66.2) & 109(71.7) & $56(78.9)$ & 0.443 \\
\hline Cancer(21) & 106(92.2) & 103(95.4) & $145(88.4)$ & 142(90.4) & 141(92.8) & 68(95.8) & 0.332 \\
\hline \multicolumn{8}{|l|}{ Positive predictive value ${ }^{g}$} \\
\hline All advanced adenomas and cancer(68) & $38(44.2)$ & $40(43.0)$ & 13(35.1) & $15(34.1)$ & $25(51.0)$ & $53(40.8)$ & 0.619 \\
\hline Cancer(21) & $12(14.0)$ & 16(17.2) & $2(5.4)$ & $6(13.6)$ & $10(20.4)$ & 18(13.8) & 0.982 \\
\hline Dukes A/B Cancer(9) & $4(4.7)$ & $6(6.5)$ & $1(2.7)$ & $3(6.8)$ & $3(6.1)$ & $7(5.4)$ & 0.810 \\
\hline Dukes C/D Cancer(12) & $8(9.3)$ & $10(10.8)$ & $1(2.7)$ & $3(6.8)$ & $7(14.3)$ & $11(8.5)$ & 0.831 \\
\hline Advanced adenomas(47) & $26(30.2)$ & $24(25.8)$ & 11(29.7) & $9(20.5)$ & $15(30.6)$ & $35(26.9)$ & 0.597 \\
\hline Advanced adenoma $\geq 10 \mathrm{~mm}(36)$ & $21(24.4)$ & $16(17.2)$ & $10(27.0)$ & $5(11.4)$ & $11(22.4)$ & $26(20.0)$ & 0.441 \\
\hline Advanced adenoma $<10 \mathrm{~mm}(11)$ & $5(5.8)$ & $8(8.6)$ & $1(2.7)$ & $4(9.1)$ & $4(8.2)$ & $9(6.9)$ & 0.746 \\
\hline
\end{tabular}

a TF combined with IFOBT (Either/or) vs IFOBT alone.

${ }^{\mathrm{b}}$ Numbers in parenthesis are the rates calculated by number of positive patients divided by the number of total participants.

c Sensitivity is the number of true positives relative to the number of persons with lesions.

$d \geq 1$ villous adenoma or adenoma with high-grade dysplasia and no adenomas $\geq 10 \mathrm{~mm}$

e Specificity is the number of true negatives relative to the number of persons without lesions. Numbers presented are the number of false-positives per group.

${ }^{f} \mathrm{NPV}$ is the number of true negatives relative to the number of persons with negative tests. Numbers presented are the number of true-negatives per group.

${ }^{g} \mathrm{PPV}$ is the number of true positives relative to the number of total positive patients.

Table 4: Results of IFOBT and TF, either alone or combination, and subsequent colonoscopic findings in symptomatic population (201 participants), n(\%).

\begin{tabular}{|c|c|c|c|c|c|c|}
\hline & Men $(n=153)$ & Women $(n=48)$ & $P$ value & Age $<60(n=75)$ & Age $\geq 60(n=126)$ & $P$ value \\
\hline \multicolumn{7}{|c|}{ Positive patients ${ }^{a}$} \\
\hline IFOBT & $67(43.8)$ & 19(39.6) & 0.607 & $28(37.3)$ & $58(46.0)$ & 0.228 \\
\hline TF & $64(41.8)$ & $29(60.4)$ & 0.024 & $37(49.3)$ & $56(44.4)$ & 0.501 \\
\hline BOTH & $32(20.9)$ & $17(35.4)$ & 0.041 & $19(25.3)$ & $30(23.8)$ & 0.808 \\
\hline EITHER & $99(64.7)$ & $31(64.6)$ & 0.988 & 46(61.3) & $84(66.7)$ & 0.444 \\
\hline \multicolumn{7}{|c|}{ Sensitivity ${ }^{b}$} \\
\hline \multicolumn{7}{|c|}{ All advanced adenomas and cancer } \\
\hline Total & 51 & 17 & & 24 & 44 & \\
\hline IFOBT & $30(58.8)$ & $8(47.1)$ & 0.398 & $12(50.0)$ & $26(59.1)$ & 0.471 \\
\hline TF & $30(58.8)$ & $10(58.8)$ & 0.776 & $16(66.7)$ & $24(54.5)$ & 0.332 \\
\hline BOTH & 19(37.3) & $6(35.3)$ & 0.885 & $10(41.7)$ & $15(34.1)$ & 0.536 \\
\hline EITHER & $41(80.4)$ & $12(70.6)$ & 0.398 & $18(75.0)$ & $35(79.5)$ & 0.666 \\
\hline \multicolumn{7}{|l|}{ Cancer } \\
\hline Total & 13 & 8 & & 11 & 10 & \\
\hline IFOBT & $9(69.2)$ & $3(37.5)$ & 0.154 & $7(63.6)$ & $5(50.0)$ & 0.528 \\
\hline TF & $10(76.9)$ & $6(75.0)$ & 0.920 & $8(72.7)$ & $8(80.0)$ & 0.696 \\
\hline BOTH & $7(53.8)$ & $3(37.5)$ & 0.466 & $6(54.5)$ & $4(40.0)$ & 0.505 \\
\hline EITHER & 12(92.3) & $6(75.0)$ & 0.271 & $9(81.8)$ & 9(90.0) & 0.593 \\
\hline
\end{tabular}

a Numbers in parenthesis are the rates calculated by number of positive patients divided by the number of total patients per group.

${ }^{b}$ Sensitivity is the number of true positives relative to the number of persons with lesions.

Table 5: Results of tests and subsequent colonoscopic findings in symptomatic population stratified by gender and age, $\mathrm{n}(\%)$.

digestive enzymes and bacteria. Therefore, transferrin is potentially a more sensitive and stable biological marker than hemoglobin. Previous proteomic studies identified transferrin as a probable protein marker for a number of malignant tumors [10,15]. Our preliminary study involving 110 patients including cancer, precancerous adenoma, and non-cancer symptomatic control showed that TF dipstick combined with IFOBT could also potentially increase the detection of colorectal cancer and advanced adenomas [11].

Consistent with the results of the previous preliminary study, our current prospective study in either the asymptomatic or symptomatic 
Citation: Jin P, Wu ZT, Meng MM, Wang X, Wang XW, et al. (2012) Combined Fecal Transferrin Test and Immuno Fecal Occult Blood Test for Detecting Colorectal Cancer and Advanced Adenoma in Asymptomatic and Symptomatic Populations. J Cancer Sci Ther 4: $243-248$. doi:10.4172/1948-5956.1000149

\begin{tabular}{|c|c|c|c|}
\hline & IFOBT & IFOBT + TF (EITHER/or) & $P$ value $^{\mathrm{c}}$ \\
\hline \multicolumn{4}{|c|}{ Patients with changes in bowel habit $(n=127)$} \\
\hline \multicolumn{4}{|c|}{ All advanced adenomas and cancer(53) } \\
\hline Sensitivity a & $30(56.6)$ & $41(77.4)$ & 0.023 \\
\hline Specificity ${ }^{b}$ & $37(50.0)$ & $50(32.4)$ & 0.030 \\
\hline \multicolumn{4}{|c|}{ Patients with bloody stool $(n=89)$} \\
\hline \multicolumn{4}{|c|}{ All advanced adenomas and cancer(40) } \\
\hline Sensitivity ${ }^{a}$ & $27(67.5)$ & $36(90.0)$ & 0.014 \\
\hline Specificity ${ }^{b}$ & $29(40.8)$ & $35(28.6)$ & 0.203 \\
\hline \multicolumn{4}{|c|}{ Patients with abdominal pain $(n=74)$} \\
\hline \multicolumn{4}{|c|}{ All advanced adenomas and cancer(22) } \\
\hline Sensitivity a & 13(59.1) & $20(90.9)$ & 0.015 \\
\hline Specificity ${ }^{b}$ & $7(86.5)$ & $24(53.8)$ & 0.000 \\
\hline
\end{tabular}

a Sensitivity is the number of true positives relative to the number of persons with lesions. Numbers presented are the number of true positives per group.

b Specificity is the number of true negatives relative to the number of persons without lesions. Numbers presented are the number of false-positives per group.

c TF combined with IFOBT (Either/or) vs IFOBT alone.

Table 6: Results of tests and subsequent colonoscopic findings in symptomatic population stratified by clinical features, $\mathrm{n}(\%)$.

population demonstrated that combining TF with IFOBT improved the detection of colorectal cancers and advanced adenomas over IFOBT alone. As expected, such an approach resulted in substantial loss of specificity, with the combined test with and/or scenario showing an overall positivity of $14.5 \%$ in the asymptomatic population and $64.7 \%$ in the symptomatic population. It should be noted, however, that positivity for the two tests appeared to be mutually exclusive in a high number of subjects with carcinomas or advanced adenomas. In the asymptomatic group, only $4.1 \%$ subjects showed positive results in both tests, and about $10 \%$ of the cases showed discordance between the two tests (Table 1). Even in the symptomatic group, only $24 \%$ subjects showed simultaneous IFOBT and TF positivity. When used alone, the two tests showed similar colonoscopic findings. Together, these results suggest that the two tests may be complementary, which provide the rationale to combine the two tests in colorectal cancer screening, as the main focus of cancer screening is to detect as many cancers and precancerous lesions as possible. It should be also noted that the two tests, either alone or in combination, will not likely replace the need of colonoscopy to evaluate the patients who present with these symptoms. However, the value of a combined TF with IFOBT is to increase the sensitivity of the detection of advanced adenomas and cancer through identifying additional cases that require colonoscopic examination relative to IFOBT alone. This is particularly true in places where medical resources may be limited (e.g., China), as the first line of work up is usually stool based analysis.

It is interesting to note that, in symptomatic group, there were 6 TF-only positive carcinomas, and only 2 IFOBT- only positive carcinomas, even though overall the positivity rates for significant lesions (carcinoma plus advanced adenoma) appear to be similar between TF-only positive group and IFOBT-only positive group. The exact reason of this phenomenon is unclear, but may be due to the "zone" effect of IFOBT, i.e., when there is a presence of large amount of bleeding, a false negative IFOBT result may be displayed whereas TF results may not be affected by this condition.

As discussed above, the addition of TF might result in more "false" positive findings. While one may always argue that some of these may represent "false" false positive findings (i.e., developing significant gastrointestinal tumors that may show up with time), there is no doubt that some of these are true false positives. One potential source of the false-positive results may be presence of the upper gastrointestinal tract lesions which are not detectable by colonoscopy, as transferrin is much more stable than hemoglobin. Another source of false-positivity may be from the urinary tract, which could partly explain the higher positive rate of TF in females. However this study did not examine either the upper gastrointestinal tract or urinary tract. We are currently investigating these possibilities in a separate study.

It should also be noted that our study had a slightly higher IFOBT positive rate in asymptomatic screen population relative to other reports ( $8 \%$ in our study versus 4 to $6 \%$ in other studies) $[5,6]$. The exact reason for that again is unclear. In asymptomatic population, not all test positive subjects received colonoscopy. We found those who refused colonoscopy were more women and younger subjects. This might result in potential bias in the estimation of the differences of efficacy in detecting advanced adenomas and cancer with different testing regimes. Another limitation of the current study is that we only examined a one-time point sensitivity and specificity. The programbased efficacy (e.g., annual screen for 5 year) of such a combined approach versus IFOBT alone remains to be determined. The key question is whether the combined approach (IFOBT and TF either/ or) provides sufficient negative predictive value so that colonoscopy can be avoided for the double negative cases. Our finding of NPV in symptomatic population (78.9\% for advanced adenoma and cancer and $95.8 \%$ for cancer) may provide support for such a notion but the ultimate determination will need to be determined in asymptomatic screening population. A prospective randomized trial will be needed to address this important issue, and our current findings provide data for supporting such a study.

In summary, our current study shows that a combination of stoolbased TF with IFOBT tests may be a viable and effective approach for colorectal cancer screening. Such a combination may increase the rate of detection of colorectal cancers and precancerous lesions with the trade off of more false positive findings. Large-scale and randomized trials are warranted to further evaluate the efficacy, especially programbased sensitivity and specificity of the combined approach.

\section{Acknowledgement} BJZ07.

This study was supported by the Zonghou Medical Foundation, Grant No.

\section{References}

1. Li LD, Rao KQ (2000) The Cancer incidence and mortality statistics of 11 cancer registries in china, 1988 - 1992. China Cancer 9: 435-447.

2. Zhang J, Dhakal IB, Zhao Z, Li L (2012) Trends in mortality from cancers of the breast, colon, prostate, esophagus, and stomach in East Asia: role of nutrition 
Citation: Jin P, Wu ZT, Meng MM, Wang X, Wang XW, et al. (2012) Combined Fecal Transferrin Test and Immuno Fecal Occult Blood Test for Detecting Colorectal Cancer and Advanced Adenoma in Asymptomatic and Symptomatic Populations. J Cancer Sci Ther 4: $243-248$. doi:10.4172/1948-5956.1000149

transition. Eur J Cancer Prev 21: 480-489.

3. Sung JJ, Lau JY, Young GP, Sano Y, Chiu HM, et al. (2008) Asia Pacific consensus recommendations for colorectal cancer screening. Gut 57: 11661176 .

4. van Rossum LG, van Rijn AF, Verbeek AL, van Oijen MG, Laheij RJ, et al (2011) Colorectal cancer screening comparing no screening, immunochemical and guaiac fecal occult blood tests: a cost-effectiveness analysis. Int J Cancer 128: 1908-1917.

5. Morikawa T, Kato J, Yamaji Y, Wada R, Mitsushima T, et al. (2005) A comparison of the immunochemical fecal occult blood test and total colonoscopy in the asymptomatic population. Gastroenterology 129: 422-428.

6. Allison JE, Sakoda LC, Levin TR, Tucker JP, Tekawa IS, et al. (2007) Screening for colorectal neoplasms with new fecal occult blood tests: update on performance characteristics. J Natl Cancer Inst 99: 1462-1470.

7. Aisen P, Listowsky I (1980) Iron transport and storage proteins. Annu Rev Biochem 49: 357-393.

8. Chiang $\mathrm{CH}$, Jeng JE, Wang WM, Jheng BH, Hsu WT, et al. (2006) A comparative study of three fecal occult blood tests in upper gastrointestinal bleeding. Kaohsiung J Med Sci 22: 223-228.

9. Yang M, Cong Y, Zhang Z, Dia C, Kong S, et al. (2003) Simultaneous determination of both transferrin and hemoglobin in the patient with digestive tract bleeding. Chin J Clin Lab Sci 21: 83-84.
10. Ward DG, Suggett N, Cheng Y, Wei W, Johnson H, et al. (2006) Identification of serum biomarkers for colon cancer by proteomic analysis. Br J Cancer 94 1898-1905.

11. Sheng JQ, Li SR, Wu ZT, Xia CH, Wu X, et al. (2009) Transferrin dipstick as a potential novel test for colon cancer screening: a comparative study with immuno fecal occult blood test. Cancer Epidemiol Biomarkers Prev 18: 2182 2185.

12. Li S, Zhang C, Xu E (1995) Supplementary value of fecal sequential occult blood test and microalbumin on improvement of the screening rate of colorectal tumors. Chin J Oncol 17: 381-383.

13. Kronborg O, Ugstad M, Fuglerud P, Johne B, Hardcastle J, et al. (2000) Faecal calprotectin levels in a high risk population for colorectal neoplasia. Gut 46 : 795-800.

14. Imperiale TF, Ransohoff DF, Itzkowitz SH, Turnbull BA, Ross ME; Colorectal Cancer Study Group (2004) Fecal DNA versus fecal occult blood for colorectalcancer screening in an average-risk population. N Engl J Med 351: 2704-2714.

15. Ahmed N, Oliva KT, Barker G, Hoffmann P, Reeve S, et al. (2005) Proteomic tracking of serum protein isoforms as screening biomarkers of ovarian cancer Proteomics 5: 4625-4636. 